ORLICZ CENTENARY VOLUME

BANACH CENTER PUBLICATIONS, VOLUME 64

INSTITUTE OF MATHEMATICS

POLISH ACADEMY OF SCIENCES

WARSZAWA 2004

\title{
LATTICE STRUCTURES IN ORLICZ SPACES
}

\author{
FRANCISCO L. HERNÁNDEZ \\ Department of Mathematical Analysis, Faculty of Mathematics \\ Madrid Complutense University, 28040 Madrid, Spain \\ E-mail: pacoh@mat.ucm.es
}

1. Introduction. This paper is a survey on some lattice and symmetric structure properties of Orlicz function spaces and rearrangement invariant (r.i.) Banach function spaces. Our main goals are to present separable Orlicz function spaces $L^{F}[0,1]$ containing isomorphically scales of $L^{p}$-spaces for $p>2$, to study lattice-isomorphic embeddings of $L^{p}$-spaces into separable r.i. function spaces on $[0,1]$ (for $1<p<\infty$ ) and to describe properties of the set $P_{F}$ of scalars $p$ such that $L^{p}$ embeds lattice-isomorphically into a separable Orlicz function space $L^{F}[0,1]$. The first part of the paper reviews several central results on the isomorphic theory of Orlicz spaces. Some open questions are included.

It is a well-known fact that several lattice and structure properties of Orlicz spaces can be described in terms of the Matuszewska-Orlicz ([M-O]) indices of Orlicz functions. These indices and their extensions are also a powerful tool in other areas (compactness of operators, interpolation,... cf. [G], [K-T], [K-M-P], [K-P-S], [M], [Mu]).

The structure of separable r.i. function spaces has been studied in the Memoirs of Johnson, Maurey, Schechtman and Tzafriri [J-M-S-T] and Kalton [K 93] (also Lindenstrauss and Tzafriri [L-T 79]). While in the case $p<2$ isomorphic embeddings of the function spaces $L^{p}$ into separable r.i. function spaces on $[0,1]$ are well known and abundant (using probabilistic techniques), in the case $p>2$ there is a strong shortage of separable r.i. function spaces on $[0,1]$ containing isomorphic copies of $L^{p}$-spaces. Thus the existence of an isomorphic embedding of $L^{p}$ (for $p>2$ ) into a separable r.i. function space $E[0,1]$ implies the existence of a sublattice of the r.i. function space $E[0,1]$ which is lattice-isomorphic to $L^{p}([\mathrm{H}-\mathrm{K}])$. Only quite recently it has been found that a certain class of separable Orlicz function spaces $L^{F}[0,1]$ have lattice-isomorphic $L^{p}$-sublattices, whose construction requires some combinatorial facts ([H-R 98]). We will describe here these results explaining the connection of this topic with the study of discrete Orlicz spaces

2000 Mathematics Subject Classification: Primary 46E30.

Partially supported by DGICYT (Spain), grant PB2001-1284.

The paper is in final form and no version of it will be published elsewhere. 
$\ell^{F}(I)$ with uncountable symmetric basis, more precisely with isomorphic embeddings of $\ell^{p}(\Gamma)$-spaces into Orlicz spaces $\ell^{F}(I)$ for uncountable sets $\Gamma \subset I$ and $1<p<\infty([\mathrm{H}-\mathrm{T}]$, [H-R 95]).

We also present universality type results for Orlicz function spaces $L^{F}[0,1]$ and for discrete Orlicz spaces $\ell^{F}(I)$ with prefixed Matuszewska-Orlicz indices (Theorems 4.1 and 6.1) obtained recently with B. Rodríguez-Salinas ([H-R 03]). Recall that in the sequence case universal Orlicz sequence spaces were constructed by Lindenstrauss and Tzafriri in [L-T 72] (see below Theorem 2.1), and that the case of Orlicz function spaces on the unbounded interval $[0, \infty)$ was considered in $[\mathrm{H}-\mathrm{Ru}]$ by $\mathrm{C}$. Ruiz and the author (see Theorem 3.3).

The notation and terminology in the paper are standard and follow the monographs [L-T 77, 79]. The text is an expanded version of the talk given at the W. Orlicz Centenary Conference and Function Spaces VII (Poznań, July 2003). The author would like to express his gratitude to the Organizing Committee for the invitation and the great hospitality.

2. Orlicz sequence spaces. We start by recalling some classical results on the isomorphic structure of Orlicz sequence spaces. We concentrate on separable Banach Orlicz sequence spaces $\ell^{F}$, hence the Matuszewska-Orlicz indices of the Orlicz function $F$ at 0 satisfy $1 \leq \alpha_{F} \leq \beta_{F}<\infty$ and the canonical basis is a symmetric basis of $\ell^{F}$. The rich structure theory of Orlicz sequence spaces was developed by Lindberg ([Li]), and Lindenstrauss and Tzafriri in the early seventies ([L-T 71], [L-T 72], [L-T 73]). They introduced the associated sets

$$
E_{F, 1}=\overline{\left\{\frac{F(t x)}{F(t)}: 0<t \leq 1\right\}}
$$

and $C_{F, 1}=\overline{\operatorname{conv}}\left(E_{F, 1}\right)$, which are compact sets in $C[0,1]$. A central result in the study of the structure of Orlicz sequence spaces $\ell^{F}$, due to Lindenstrauss and Tzafriri [LT 73], is the following:

An Orlicz sequence space $\ell^{F}$ has a subspace isomorphic to another Orlicz sequence space $\ell^{G}$ if and only if the function $G$ is equivalent near 0 to a function of the set $C_{F, 1}$. In a short form: $\ell^{F} \underset{\sim}{\supset} \ell^{G} \Leftrightarrow G \in C_{F, 1}$.

In particular, for the $\ell^{p}$-spaces, $1 \leq p<\infty$, the following characterization holds:

$$
\ell^{F} \underset{\sim}{\supset} \ell^{p} \Leftrightarrow x^{p} \in C_{F, 1} \Leftrightarrow p \in\left[\alpha_{F}, \beta_{F}\right]
$$

The behavior of complemented $\ell^{p}$-subspaces in Orlicz sequence spaces is more involved. As these spaces have a symmetric basis, the natural way for getting a projection onto a subspace is by representing such a subspace as the span of a block basis with constant coefficients and using the canonical averaging projection. Thus we have:

$$
x^{p} \in E_{F, 1} \Rightarrow \ell^{F} \underset{\sim_{c}}{\supset} \ell^{p}
$$

However, this form is not the only way to do it. Kalton [K 77] showed that the converse is not true for $p>1$ : there exist Orlicz sequence spaces $\ell^{F}$ with $x^{p} \notin E_{F, 1}$ which contain a complemented $\ell^{p}$-subspace. This is obtained by constructing Orlicz sequence spaces $\ell^{F}$ 
(very close to $\ell^{p}$ ) such that $x^{p} \notin E_{F, 1}$ and the canonical inclusion $\ell^{F} \subseteq \ell^{p}$ is not a strictly singular operator, in this way we can carry the orthogonal projection defined on the space $\ell^{p}$ to the space $\ell^{F}$. Briefly: the space $\ell^{F}$ has a singular $\ell^{p}$-complemented copy.

For a fixed Orlicz sequence space $\ell^{F}$ let us denote by $P_{\ell^{F}}$ the set of scalars $p$ such that $\ell^{p}$ embeds as a complemented subspace into $\ell^{F}$, i.e.

$$
P_{\ell^{F}}:=\left\{p \geq 1: \ell^{F} \underset{\sim_{c}}{\supset} \ell^{p}\right\} \subset\left[\alpha_{F}, \beta_{F}\right]
$$

The sets $P_{\ell^{F}}$ have a varied geometry. Indeed, in many natural examples of Orlicz functions the set $P_{\ell^{F}}$ coincides with the whole interval $\left[\alpha_{F}, \beta_{F}\right]$. On the other hand, Lindenstrauss and Tzafriri [L-T 73] proved that the set $P_{\ell^{F}}$ can be empty. This happens for the class of minimal Orlicz functions at 0 (recall that a function $F$ is minimal at 0 if $E_{F, 1}=E_{G, 1}$ for every function $\left.G \in E_{F, 1}\right)$. Minimal Orlicz sequence spaces $\ell^{F}$ $\left(\neq \ell^{p}, 1 \leq p<\infty\right)$ have uncountably many mutually nonequivalent symmetric bases ([L-T 73]). Later on, in ([H-R 88]), Rodríguez-Salinas and the author showed that the set $P_{\ell^{F}}$ can be quite arbitrary, precisely it can be any closed subset of any given interval $\left[\alpha_{F}, \beta_{F}\right]$. Moreover, this can be shown by constructing Orlicz sequence spaces $\ell^{F}$ with only singular complemented copies of $\ell^{p}$ ([H-R 89]). It is an open question whether the set $P_{\ell^{F}}$ is always closed.

There are other interesting open problems concerning the structure of Orlicz sequence spaces. For instance, whether the class of strongly-minimal Orlicz functions (Kalton [K 90]) provides examples of prime Orlicz sequence spaces (recall that a Banach space $E$ is prime if every infinite-dimensional complemented subspace of $E$ is isomorphic to $E$ ). Up to now, the only known prime spaces with an unconditional basis are the spaces $c_{0}$ and $\ell^{p}$ for $1 \leq p<\infty$. It is unknown whether the Orlicz sequence spaces $\ell^{F_{p}}$ are prime, where

$$
F_{p}(x)=x^{p} \exp \left\{\sum_{n=1}^{\infty}\left(1-\cos \left(\pi \log x / 2^{n}\right)\right)\right\} .
$$

The existence of universal Orlicz sequence spaces with given indices was proved by Lindenstrauss and Tzafriri in ([L-T 72]):

TheOREM 2.1. There exist Orlicz sequence spaces $\ell^{F}$ with given indices $1 \leq \alpha<\beta<\infty$, such that every Orlicz sequence space $\ell^{G}$, with indices between $\alpha$ and $\beta$ is isomorphic to a complemented subspace of the space $\ell^{F}$ (more precisely $G \in E_{F, 1}$ ).

There is uniqueness of these universal Orlicz sequence spaces $\ell^{F}$ with given indices. This follows easily from the complementation (using the Pełczynski decomposition method). These universal spaces $\ell^{F}$ provide interesting examples of Banach spaces with a symmetric basis, different from $\ell^{2}$, which are isomorphic to their dual spaces (taking $\alpha$ and $\beta$ to be conjugated indices, i.e. $1 / \alpha+1 / \beta=1$ ).

3. Orlicz function spaces on $[0,1]$. We now consider separable Orlicz function spaces $L^{F}[0,1]$. The study of the structure of Orlicz function spaces was initiated by Lindenstrauss and Tzafriri in [L-T 73] introducing suitable $E_{F, 1}^{\infty}, C_{F, 1}^{\infty}$ sets and using the KadecPełczyński disjointification method. The characterization of $\ell^{p}$-subspaces is the following:

$$
L^{F}[0,1] \underset{\sim}{\supset} \ell^{p} \Leftrightarrow p \in\left[\alpha_{F}^{\infty}, \beta_{F}^{\infty}\right] \cup\left(\beta_{F}^{\infty}, 2\right) \cup\{2\},
$$


where $\alpha_{F}^{\infty}$ and $\beta_{F}^{\infty}$ denote the Matuszewska-Orlicz indices of the function $F$ at $\infty$. The case $p=2$ is clear (using the Rademacher functions and Khintchine inequality). And the case of $p$ belonging to the interval $\left(\beta_{F}^{\infty}, 2\right)$ is obtained via $p$-stable random variables.

For a fixed Orlicz function space $L^{F}[0,1]$, we consider the set $P_{L^{F}}$ of $p$ 's such that $L^{F}[0,1]$ has a complemented $\ell^{p}$-subspace, i.e.

$$
P_{L^{F}}:=\left\{p \geq 1: L^{F}[0,1] \underset{\sim_{c}}{\supset} \ell^{p}\right\}
$$

The geometry of the sets $P_{L^{F}}$ can be varied. It is clear, by duality, that $P_{L^{F}} \subset\left[\alpha_{F}^{\infty}, \beta_{F}^{\infty}\right] \cup$ $\{2\}$. The existence of Orlicz function spaces $L^{F}[0,1]$ where the set $P_{L^{F}}$ is the singleton $\{2\}$ was proved by Peirats and the author [H-P] by introducing minimal Orlicz function spaces. Later on in $[\mathrm{H}-\mathrm{R} 88,89]$ it was proved that the set $P_{L^{F}}$ can be any closed subset of the interval $\left[\alpha_{F}^{\infty}, \beta_{F}^{\infty}\right]$ union $\{2\}$. Furthermore this can be done either with natural $\ell^{p}$-copies (averaging projections) or with singular $\ell^{p}$-copies. It is unknown whether the set $P_{L^{F}}$ is always closed. Other distinguished classes of complemented subspaces in Orlicz function spaces $L^{F}[0,1]$ have been studied in e.g. [Ru], [Ra] and [A-M-S].

We concentrate now on discussing isomorphic embeddings of the function spaces $L^{p}[0,1]$ into separable Orlicz function spaces $L^{F}[0,1]$ and, more general, into separable r.i. function spaces $E[0,1]$ (i.e. $L^{F}[0,1] \underset{\sim}{\supset} L^{p}$ and $E[0,1] \underset{\sim}{\supset} L^{p}$ ). Note that there is no ambiguity in this short notation since $L^{p}$-spaces on $[0,1]$ and on $[0, \infty)$ are lattice-isomorphic. A systematic study of the symmetric structure of r.i. function spaces, in particular Orlicz spaces, was carried out in the Memoir of Johnson, Maurey, Schechtman and Tzafriri [J-M-S-T]. A remarkable predecessor was the work of Bretagnolle and Dacunha-Castelle [B-Dc]. Other important contributions are due to Kalton ([K 79], [K 93]). We present here a sample of some representative results chosen by their relevance to our purposes.

It turns out that the structure of $r$.i. function spaces on $[0,1]$ is quite more rigid than those in the sequence case or in the case of function spaces on the unbounded $[0, \infty)$ interval. Thus ([J-M-S-T]):

THEOREM 3.1. Let E[0,1] be an r.i. function space which does not contain isomorphic copies of $\ell_{n}^{\infty}$ uniformly and $F[0,1]$ be a separable r.i. function space $\left(\neq L_{2}\right)$ with nontrivial indices. If $E[0,1] \underset{\sim}{\supset} F[0,1]$ then either $E[0,1] \supset F[0,1]$ or the Haar basis of $F[0,1]$ is equivalent to a disjoint sequence in $E[0,1]$.

In particular for Orlicz spaces $L^{F}[0,1]$, due to the impossibility of embedding isomorphically Orlicz function spaces into sequence spaces, we have that $L^{F}[0,1] \underset{\sim}{\supset} L^{G}[0,1]$ $\left(\neq L^{2}\right) \Rightarrow L^{F}[0,1] \supset L^{G}[0,1]$. This inclusion property obtained from the existence of an isomorphic embedding is also true when we have disjointness preserving operators between order continuous r.i. function spaces on $[0,1]$ (in particular for lattice homomorphisms [A]). It follows that reflexive Orlicz function spaces on $[0,1]$ have a unique representation as Orlicz function spaces:

$$
L^{F}[0,1] \approx L^{G}[0,1] \Rightarrow L^{F}[0,1]=L^{G}[0,1]
$$

In general all separable Orlicz function spaces on $[0,1]$ have a unique structure as r.i. function spaces on $[0,1]$ (see the recent survey of Tzafriri $[\mathrm{Tz}]$ ). 
On the other hand, an Orlicz function space $L^{F}[0,1]$ cannot contain complemented copies of other Orlicz function spaces: if $L^{F}[0,1] \underset{\sim_{c}}{\supset} L^{G}[0,1]\left(\neq L^{2}\right)$ then $L^{F}[0,1]=$ $L^{G}[0,1]$. Moreover, reflexive Orlicz function spaces $L^{F}[0,1]$ are primary spaces (i.e. every decomposition of $L^{F}[0,1]$ into a direct sum of two closed subspaces has at least one of the factors isomorphic to $\left.L^{F}[0,1]\right)$.

A general result of Kalton [K 93] (obtained earlier under some extra conditions in [K 79] and also in [J-M-S-T]) claims that a separable r.i. function space E[0,1] contains an isomorphic copy of $L^{1}$ if and only if $E[0,1]=L^{1}[0,1]$, up to an equivalent renorming. This is obtained in two steps: first, a separable r.i. function space $E[0,1]$ containing an isomorphic copy of $L^{1}$ must also contain a lattice-isomorphic copy of $L^{1}$; from this it is deduced next that $E[0,1]=L^{1}[0,1]$ up to an equivalent renorming, i.e.

$$
E[0,1] \underset{\sim}{\supset} L^{1} \Rightarrow E[0,1] \underset{\sim}{\supset} L^{1} \Rightarrow E[0,1]=L^{1}[0,1]
$$

We will present several extensions of these statements, replacing the space $L^{1}$ by $L^{p}$ for $1<p<\infty$ under some suitable conditions.

A useful criterion for the lattice-embedding of function spaces into r.i. function spaces $E(I)$ over an interval $I$ was given in ([J-M-S-T]). Let $g$ be a positive normalized function, we can consider the sublattice generated by the function $g$, defined by $E_{g}(I)=\{f$ : $f \times g \in E(I \times I)\}$ since $E(I \times I)$ is lattice isomorphic to $E(I)$. In the case of Orlicz function spaces $E(I)=L^{F}(I)$ it turns out that the sublattice $\left(L^{F}\right)_{g}(I)$ coincides with an Orlicz function space $L^{F_{g}}(I)$ associated to a certain Orlicz function $F_{g}$. Let us denote by $\sum_{F, 1}^{\infty}$ the set of Orlicz functions $G$ of the form

$$
G(x) \cong \int_{0}^{\infty} \frac{F(s x)}{F(s)} d \mu(s)
$$

for $x>1$, where $\mu$ is a probability measure on $(0, \infty)$ such that $\int_{0}^{\infty} \frac{1}{F(s)} d \mu(s) \leq 1$. Thus we have the following ([J-M-S-T] Thm 7.7):

TheOREM 3.2. Given a separable Orlicz function space $L^{F}[0,1]$, if $G \in \sum_{F, 1}^{\infty}$ then $L^{F}[0,1]$ $\supset_{\sim_{\ell}} L^{G}[0,1]$. Furthermore, if $L^{F}[0,1]$ is $p$-convex for some $p>2$ and $E[0,1]\left(\neq L^{2}\right)$ is an r.i. function space which embeds isomorphically into $L^{F}[0,1]$, then $E[0,1]=L^{G}[0,1]$, up to an equivalent renorming, for some Orlicz function $G \in \sum_{F, 1}^{\infty}$.

In general, the sets $\sum_{F, 1}^{\infty}$ are noncompact and that is an obstacle when looking inside them for a concrete function.

Universal Orlicz function spaces on $[0, \infty)$. Before we go any further let us briefly mention some results for function spaces on $[0, \infty)$, showing the abundance of separable r.i. function spaces on $[0, \infty)$ containing $L^{p}$-sublattices $(1<p<\infty)$.

The structure of separable Orlicz function spaces $L^{F}[0, \infty)$ has several peculiar and interesting properties which have been studied in [J-M-S-T], [N], [H-R 92], [K 93] and [H$\mathrm{S}]$. For example, the characterization of $\ell^{p}$-subspaces in terms of the Matuszewska-Orlicz indices of the function at 0 and at $\infty$ is now:

$$
L^{F}[0, \infty) \underset{\sim}{\supset} \ell^{p} \Leftrightarrow p \in\left[\alpha_{F}, \beta_{F}\right] \cup\left[\alpha_{F}^{\infty}, \beta_{F}^{\infty}\right] \cup\left(\beta_{F}^{\infty}, 2\right) \cup\{2\} \cup\left(\beta_{F}^{\infty}, \alpha_{F}\right) .
$$


Universal Orlicz function spaces $L^{F}[0, \infty)$ with prescribed indices were given by C. Ruiz and the author in [H-Ru]: the classical spaces $L^{\alpha}+L^{\beta}$, regarded as the Orlicz function spaces $L^{x^{\alpha} \wedge x^{\beta}}[0, \infty)$, are universal in the following sense:

TheOREm 3.3. Given $1 \leq \alpha<\beta<\infty$, the space $L^{\alpha}+L^{\beta}$ is lattice universal for the class of all Orlicz function spaces $L^{G}[0, \infty)$ with indices strictly between $\alpha$ and $\beta$, i.e.

$$
L^{\alpha}+L^{\beta} \underset{\sim}{\supset} L^{G}[0, \infty) .
$$

In particular $L^{\alpha}+L^{\beta} \underset{\sim}{\supset} L^{p}$ for every $\alpha<p<\beta$. The embedding behavior is varied in the extreme cases of Orlicz spaces $L^{G}[0, \infty)$ with one of their indices equal to $\alpha$ or $\beta$. For example if $\alpha<2<\beta$ or if $2<\alpha<\beta$, the space $L^{\beta}$ is not isomorphic to a subspace of $L^{\alpha}+L^{\beta}([\mathrm{G}-\mathrm{H}],[\mathrm{H}-\mathrm{K}])$

The proof uses some "interpolation" arguments connecting the behavior of a function near 0 and near $\infty$ in order to represent every Orlicz function $G$ in an integral form with respect to the function $x^{\alpha} \wedge x^{\beta}$, and some ideas from the works [B-Dc] and [J-M-S-T] (also [D 90] for the special scale $L^{2}+L^{p}$ ).

Returning to [0,1], the study of isomorphic embeddings of $L^{p}$-spaces into separable r.i. function spaces on $[0,1]$ leads to distinguish essentially two different cases: the 2-concave case and the opposite.

The 2-concave case on $[0,1]$. In the 2-concave case there is a large amount of separable r.i. function spaces containing isomorphically scales of $L^{p}$-spaces for $p \leq 2$. This is a wellknown fact and requires some probabilistic tools (Poisson process, or $p$-stable variables and ultrapowers). Thus ([J-M-S-T] Section 8, [L-T 79] p. 212):

Let $E[0,1]$ be an r.i.function space. If the function $x^{-1 / p} \in E[0,1]$ for some $1<p<2$, then $E[0,1] \underset{\sim}{\supset} L^{p}$ (isometrically).

In particular for $1 \leq q \leq p \leq 2$, we have $L^{q}[0,1] \supset L^{p}$ (isometrically), a classical result of Bretagnolle, Dacunha-Castelle and Krivine [B-Dc-K].

Numerous authors have carefully analyzed which other classical function spaces with (and without) symmetric structure can be isomorphically embedded into the spaces $L^{q}[0,1]$ for $1 \leq q<2$. For example for the class of Orlicz function spaces we have : $L^{1}[0,1] \underset{\sim}{\supset} L^{F}[0,1]$ if and only if the Orlicz space $L^{F}[0,1]$ is 2-concave (Bretagnolle and Dacunha-Castelle [B-Dc], Schütt [S 95]). For the Lorentz function space class, similar results were obtained by Schütt in [S 89] (see also Raynaud and Schütt [R-S], the recent survey of Dilworth [D 01] and the references there).

The non-2-concave case on $[0,1]$. In contrast with the above case, there is a strong shortage of separable r.i. function spaces on $[0,1]$ containing isomorphically scales of $L^{p}$ spaces, for $p>2$. Recall for example that for the spaces $L^{q}[0,1]$ (with $q>2$ ), the Banach lattices that can be isomorphically embedded into $L^{q}[0,1]$ are essentially $L^{q}(\mu)$-spaces for some suitable measures $\mu$ (cf. [L-T 79] p. 202). In particular, the r.i. function spaces on $[0,1]$ which are isomorphic to a subspace of $L^{q}[0,1]$, for $q>2$, are just $L^{q}[0,1]$ or $L^{2}[0,1]$ ([J-M-S-T] p. 41, [Tz]). 
Given any $2<p<\infty$, for quite a time no example was known of a separable r.i. function space $E[0,1]\left(\neq L^{p}[0,1]\right)$ such that $E[0,1] \underset{\sim}{\supset} L^{p}$. The impossibility of finding such examples inside the class of Lorentz function spaces was showed by Carothers ([C 81, 87]). In [H-R 95], Rodríguez-Salinas and the author constructed the first (nontrivial) examples of separable r.i. function spaces (indeed Orlicz spaces $L^{F}[0,1]$ ) containing an isomorphic copy of $L^{p}$ for a given $p>2$. This shortage of r.i. function spaces is related to the following fact proved by Kalton and the author in ([H-K] p. 827):

THEOREM 3.4. Let $E[0,1]$ be a r.i.function space with some concavity and p-convex for some $p>2$. If a r.i. function space $F[0,1]\left(\neq L^{2}\right)$ is isomorphic to a subspace of $E[0,1]$ then $F[0,1]$ is lattice-isomorphic to a sublattice of $E[0,1]$.

In particular, the existence of an isomorphic embedding of an $L^{p}$-space for $p>2$ into a separable r.i. function space $E[0,1]$ implies that there exists also a lattice-isomorphic embedding of $L^{p}$ into $E[0,1]$ ( i.e. for $2<p<\infty, E[0,1] \underset{\sim}{\supset} L^{p} \Rightarrow E[0,1] \underset{\sim_{\ell}}{\supset} L^{p}$ ).

This phenomenon occurs also in other situations. Thus for Banach lattices which are complemented subspaces of separable r.i. function spaces $([\mathrm{H}-\mathrm{K}]$ p. 831$)$ : Let $E[0,1]$ be a separable r.i. function space which contains no complemented sublattice isomorphic to $\ell^{2}$. If a p-convex Banach lattice $F$, for some $p>2$, is isomorphic to a complemented subspace of $E[0,1]$ then $F$ is lattice-isomorphic to a complemented sublattice of $E[0,1]$.

We finish this section by mentioning that Kalton's result on isomorphic $L^{1}$-embeddings (given in Section 3) can be extended to isomorphic $L^{p}$-embeddings for $p>2$ under some extra conditions $([\mathrm{H}-\mathrm{K}]$ p. 828$)$.

THEOREM 3.5. (i) If a p-convex $(p>2)$ r.i. function space $E[0,1]$, with some concavity contains an isomorphic copy of $L^{p}$, then $E[0,1]=L^{p}[0,1]$, up to an equivalent renorming.

(ii) If a p-concave r.i. function space $E[0,1]$ which is also $r$-convex (for some $r>2$ ), contains an isomorphic copy of $L^{p}$, then $E[0,1]=L^{p}[0,1]$, up to an equivalent renorming.

4. Universal Orlicz function spaces $L^{F}[0,1]$. The existence of separable Orlicz function spaces $L^{F}[0,1]$ containing lattice-isomorphically scales of $L^{p}$-spaces for different values of $p$ has been proved in [H-R9 8]. In particular for scalars $p>2$ this provided the first examples of separable r.i. function spaces on $[0,1]$ containing isomorphically scales of $L^{p}$-spaces. A more general result on universality given recently in [H-R 03] is the following:

THEOREM 4.1. Given $1<\alpha<\beta<\infty$, there exists an Orlicz function space $L^{F_{\alpha, \beta}}[0,1]$, with indices $\alpha_{F_{\alpha, \beta}}^{\infty}=\alpha$ and $\beta_{F_{\alpha, \beta}}^{\infty}=\beta$, such that every $\alpha$-convex $\beta$-concave Orlicz function space $L^{G}[0,1]$ is lattice-isomorphic to a sublattice of $L^{F_{\alpha, \beta}}[0,1]$.

Thus the spaces $L^{F_{\alpha, \beta}}[0,1]$ satisfy $L^{F_{\alpha, \beta}}[0,1] \underset{\sim_{\ell}}{\supset^{G}}[0,1]$, in particular for every $\alpha \leq$ $p \leq \beta$ we have $L^{F_{\alpha, \beta}}[0,1] \underset{\sim_{\ell}}{\supset} L^{p}$.

The above universal function spaces $L^{F_{\alpha, \beta}}[0,1]$ are neither $\alpha$-convex nor $\beta$-concave (see Theorem 3.5). On the other hand, the hypothesis of $\alpha$-convexity and $\beta$-concavity of the function spaces $L^{G}[0,1]$ cannot be removed. 
The method used in the proof of last Theorem involves some combinatorial facts and properties of Banach spaces with uncountable symmetric basis. More precisely, the existence of discrete Orlicz spaces $\ell^{F}(I)$ containing isomorphic copies of $\ell^{p}(\Gamma)$-spaces for uncountable sets $\Gamma \subset I$. The following two sections are devoted to describe this.

5. Banach spaces with an uncountable symmetric basis. The structure of Banach spaces with an uncountable symmetric basis has a behavior quite different to the countable case of sequence spaces. Let us review some representative results. Recall that a family of vectors $\left(e_{i}\right)_{i \in I}$ in a Banach space $E$ is a symmetric basis if it is anconditional basis and for every pair $\left(i_{k}\right)$ and $\left(i_{j}\right)$ of sequences of different elements indices in $I$ we have that $\left(e_{i_{k}}\right)$ and $\left(e_{i_{j}}\right)$ are equivalent basic sequences.

Using renorming arguments, Troyanski [T 75] proved that: if a Banach space $E$ with an uncountable symmetric basis $\left(e_{i}\right)_{i \in I}$ contains an isomorphic copy of $\ell^{1}(\Gamma)$ for some uncountable $\Gamma \subset I$, then $E=\ell^{1}(I)$.

A similar result holds for the space $c_{0}(\Gamma)$. Namely if a Banach space $E$ with an uncountable symmetric basis verifies $E \underset{\sim}{\supset} c_{0}(\Gamma)$ for some uncountable $\Gamma \subset I$ then $E=$ $c_{0}(I)$. This was also proved by Troyanski in [T 75]. In the uncountable case, there is uniqueness of symmetric basis (Drewnowski [Dr]), in contrast with the behavior of the countable case. A special type of block basis is of interest in the study of spaces with an uncountable symmetric basis: a basic set $\left(v_{j}\right)_{j \in \Gamma}$ in a Banach space $E$ is a block basis generated by one vector $y=\sum_{n=0}^{\infty} a_{n} e_{i_{n}}$ if there exist disjoint infinite subsets $(j(k))_{k=1}^{\infty}$ in $I$, for each $j \in \Gamma$, where $j \neq l$ or $k \neq m$ implies $j(k) \neq l(m)$, such that every $v_{j}=\sum_{k=1}^{\infty} a_{k} e_{j(k)}$ for each $j \in \Gamma$. A key result is the following structure theorem due to Troyanski [T 90] (also valid for quasi-Banach spaces [H-T]):

THEOREM 5.1. Let $E$ be a Banach space with an uncountable symmetric basis. If $\left(u_{j}\right)_{j \in \Gamma}$ is an uncountable symmetric basic set in $E$, then $\left(u_{j}\right)_{j \in \Gamma}$ is equivalent to a block basis generated by one vector.

Using this it is shown that Lorentz spaces $d(w, p, I), 1 \leq p<\infty$, do not contain any isomorphic copy of $\ell^{p}(\Gamma)$ for uncountable $\Gamma \subset I$ (compare with the positive results in the sequence case cf. [L-T 77]).

A natural question is to study possible extensions of Troyanski's result on $\ell^{1}(\Gamma)$ embeddings to the case $1<p<\infty$, i.e. whether there exist Banach spaces $\left(\neq \ell^{p}(I)\right)$ with an uncountable symmetric basis containing an isomorphic copy of $\ell^{p}(\Gamma)$ for uncountable $\Gamma$. The answer is yes, and the first examples were certain non-reflexive Orlicz spaces with symmetric basis given by Troyanski and the author in [H-T].

For a fixed discrete Orlicz space $\ell^{F}(I)$, we consider the set $\sum_{F, 1}$ of all Orlicz functions

$$
G(x)=\int_{0}^{1} \frac{F(s x)}{F(s)} d \mu(s) \quad(\text { for } 0<x<1),
$$

where $\mu$ is a probability measure on $(0,1]$. The inclusion $\sum_{F, 1} \subset C_{F, 1}$ holds, and the set $\sum_{F, 1}$ is in general noncompact. Other properties of the set $\sum_{F, 1}$ are: if $G \in \sum_{F, 1}$ then the Young conjugate $G^{*} \notin \sum_{F^{*}, 1}$, and $x^{q} \sum_{F, 1} \cong \sum_{x^{q} F, 1}$ for every $q>0$. The following criterion is useful $([\mathrm{R}],[\mathrm{H}-\mathrm{T})$ : 
TheOREM 5.2. Given an Orlicz space $\ell^{F}(I)$ with the function $F$ satisfying the $\Delta_{2}$-condition at 0 . Then $\ell^{F}(I)$ contains an isomorphic copy of $\ell^{G}(\Gamma)$ for $\Gamma \subset I$ uncountable sets if and only if $G \in \sum_{F, 1}$.

6. Fundamental lemmas. In this section we indicate the methods used in the proofs of the above results. We concentrate on the existence of reflexive Orlicz function spaces $L^{G}[0,1]$ containing a lattice-isomorphic copy of $L^{p}$ with given indices $\alpha_{G}^{\infty}=\alpha$ and $\beta_{G}^{\infty}=\beta$ and $\alpha<p<\beta$.

Let us first point out that this result can be quite easily deduced after solving a related problem for the uncountable discrete case, i.e. the existence of discrete Orlicz spaces $\ell^{F}(I)$ containing an isomorphic copy of $\ell^{p}(\Gamma)$ for uncountable $\Gamma \subset I$. Indeed, by transfer arguments, we consider some $r>\beta$ and define then an Orlicz function $G$ near $\infty$ by

$$
G(x):=x^{r} F(1 / x)
$$

where $F$ is a certain Orlicz function defined near 0 such that $\ell^{F}(I) \underset{\sim}{\supset} \ell^{p}(\Gamma)$ for uncountable $\Gamma \subset I$. Using Theorem 5.2, the criterion for lattice embedding $\widetilde{L}^{p}$ spaces into Orlicz function spaces $L^{G}[0,1]$ given in terms of the set $\sum_{G, 1}^{\infty}$ (Theorem 3.2) can be applied to get the $L^{p}$-embedding.

We now focus on the construction of discrete Orlicz spaces $\ell^{F}(I) \operatorname{such}$ that $\ell^{F}(I) \underset{\sim}{\supset}$ $\ell^{p}(\Gamma)$ for uncountable $\Gamma \subset I$ and prefixed indices. A crucial point in doing this is the existence of series of positive terms with the following "shift uniformly bounded" property:

LEMmA 1. There exist sequences $\left(\alpha_{n}\right)_{n=0}^{\infty}$ and $\left(\varepsilon_{n}\right)_{n=0}^{\infty}$ of positive numbers with $\sum_{n=0}^{\infty} \alpha_{n}$ $=\infty$ and constants $A>0$ and $B>0$ such that

$$
A \leq \sum_{n=0}^{\infty} \alpha_{n} \varepsilon_{n+k} \leq B
$$

for every natural $k=0,1,2, \ldots$

The existence of these sequences $\left(\alpha_{n}\right)$ and $\left(\varepsilon_{n}\right)$ is proved using the following combinatorial fact: given an arbitrary sequence $\left(h_{i}\right)_{i=0}^{\infty}$ of positive integers with $h_{0}=1$, there exists a set of couples of positive integers $\left\{\left(m_{j}, k_{i}\right)\right\}$ with $m_{i}>k_{i}$ such that for each $n$ :

(i) there exist precisely $h_{n}$ couples $\left(m_{j}, k_{i}\right)$ such that $m_{j}-k_{i}=n$.

(ii) there exist at most $(n+2)^{2}$ couples $\left(m_{j}, k_{i}\right)$ such that $k_{i}-m_{j}=n$.

This property is precisely formulated as follows ([H-T], [H-R 95]):

LEMmA 2. Given $\left(h_{i}\right)_{i=0}^{\infty}$ a sequence of positive integers with $h_{0}=1$, there exist two positive integer sequences $\left(k_{i}\right)_{i=0}^{\infty}$ and $\left(m_{i}\right)_{i=0}^{\infty}$ with $m_{i}>k_{i}=\sum_{j=0}^{i-1} m_{j}$ for $i=1,2, \ldots$ and $\lim _{i \rightarrow \infty}\left(m_{i+1}-m_{i}\right)=\infty$, such that if $f=\sum_{i=0}^{\infty} \chi_{\left[m_{i}, m_{i}+1\right)}$ then

$$
\sum_{i=0}^{\infty} f\left(n+k_{i}\right)=h_{n}, \quad \sum_{i=0}^{\infty} f\left(k_{i}-n\right) \leq(n+2)^{2} .
$$

Let us indicate now other steps of the proof of $\ell^{F}(I) \underset{\sim}{\supset} \ell^{p}(\Gamma)$. We can assume $\alpha=$ $1<p<\beta=p+\varepsilon$. The other cases can be deduced from this using $q$-concavification and 
$r$-convexification reductions and properties of the $\sum_{F, 1}$ sets. We consider the function

$$
f=\sum_{n=0}^{\infty} \varepsilon_{n} \chi_{\left(2^{-(n+1)}, 2^{-n}\right]}
$$

where the sequence $\left(\varepsilon_{n}\right)$ is given by Lemma 1 . And define the Orlicz function $F$ at 0 by

$$
F(x)=\int_{0}^{x}(x-t) t^{p-2} f(t) d t .
$$

It turns out that the function $f$ satisfies the following key property:

$$
A \leq \sum_{n=0}^{\infty} \alpha_{n} f\left(\frac{x}{2^{n}}\right) \leq B
$$

for $0<x \leq 1$. From this, the following inequalities are obtained by integration:

$$
A \frac{x^{p}}{p(p-1)} \leq \sum_{n=0}^{\infty} \alpha_{n} 2^{p n} F\left(\frac{x}{2^{n}}\right) \leq B \frac{x^{p}}{p(p-1)}
$$

for $0 \leq x \leq 1$. Thus, if we consider the discrete measure $\mu$ on $(0,1]$ defined by $\mu\left(2^{-n}\right):=$ $\alpha_{n} 2^{p n} F\left(2^{-n}\right)$, we deduce that the function $G$, defined by

$$
G(x)=\int_{0}^{1} \frac{F(x t)}{F(t)} d \mu
$$

satisfies that $x^{p} \cong G \in \sum_{F, 1}$. Hence, using Theorem 5.2, we deduce that the Orlicz space $\ell^{F}(I)$ verifies $\ell^{F}(I) \underset{\sim}{\supset} \ell^{p}(\Gamma)$ for uncountable $\Gamma \subset I$.

Finally, using properties of the sequence $\left(\varepsilon_{n}\right)$ constructed in Lemma 1, the associated indices of the Orlicz function $F$ can be computed to obtain $\alpha_{F}=1$ and $\beta_{F}=p+\varepsilon$.

A more general result is the existence of universal Orlicz spaces $\ell^{F}(I)$ which is proved using a similar technique ([H-R 03]):

TheOrem 6.1. Let $1<\alpha<\beta<\infty$. There exists an Orlicz space $\ell^{F_{\alpha, \beta}}(I)$, with indices $\alpha_{F_{\alpha, \beta}}=\alpha$ and $\beta_{F_{\alpha, \beta}}=\beta$, such that $\ell^{F_{\alpha, \beta}}(I)$ contains an isomorphic copy of any $\alpha$-convex $\beta$-concave Orlicz space $\ell^{G}(\Gamma)$ with $\Gamma \subset I$ arbitrary sets.

There is no uniqueness up to isomorphism of these universal spaces $\ell^{F_{\alpha, \beta}}(I)$ as in the sequence case (see Theorem 2.1). The above theorem provides also new examples of universal Orlicz sequence spaces $\ell^{F_{\alpha, \beta}}$ with given indices $\alpha$ and $\beta$ different from those given by Lindenstrauss and Tzafriri [L-T 72]. Indeed these universal sequence spaces $\ell^{F_{\alpha, \beta}}$ do not have any complemented copy of other sequence spaces $\ell^{G}$.

7. Properties of the sets $P_{F}$. Given a separable Orlicz function space $L^{F}[0,1]$, let us consider now the associated set $P_{F}$ of scalars $p$ such that $L^{p}$ embeds lattice isomorphically into $L^{F}[0,1]$, i.e.

$$
P_{F}:=\left\{p>1: L^{F}[0,1] \underset{\sim_{\ell}}{\supset_{\ell}} L^{p}\right\}
$$

In this last section we present some properties of these sets $P_{F}$. To this end, it is useful to consider a third parameter (different from the Matuszewska-Orlicz indices): the 
"inclusion" index $\gamma_{F}^{\infty}$ associated to $F$. Define

It turns out that

$$
\gamma_{F}^{\infty}:=\limsup _{x \rightarrow \infty} \frac{\log F(x)}{\log x} .
$$

$$
\gamma_{F}^{\infty}=\inf \left\{p>1: L^{p}[0,1] \subset L^{F}[0,1]\right\}
$$

It is easy to check that $\alpha_{F}^{\infty} \leq \gamma_{F}^{\infty} \leq \beta_{F}^{\infty}$. And these inequalities can be strict. Clearly if $p$ is a scalar such that $L^{F}[0,1] \underset{\sim_{\ell}}{\supset} L^{p}$, then $\gamma_{F}^{\infty} \leq p \leq \beta_{F}^{\infty}$. Hence

$$
P_{F} \subset\left[\gamma_{F}^{\infty}, \beta_{F}^{\infty}\right] \text {. }
$$

Many natural Orlicz functions $F$ are such that their associated set $P_{F}$ is just the empty set: for example the class of submultiplicative functions at $\infty$. On the other hand notice that the universal Orlicz function spaces $L^{F_{\alpha, \beta}}[0,1]$, given in Theorem 4.1, have inclusion index $\gamma_{F_{\alpha, \beta}}^{\infty}=\alpha_{F_{\alpha, \beta}}^{\infty}$ and that in this case the sets $P_{F}$ reach their biggest possible size filling all of the interval $[\alpha, \beta]$, i.e. $P_{F}=[\alpha, \beta]$.

The following result shows in particular that the "size" of the sets $P_{F}$ can be arbitrarily small comparing with the size of the interval $[\alpha, \beta]([\mathrm{H}-\mathrm{R} 98])$ :

TheOREM 7.1. Let $1<\alpha<\gamma \leq \beta<\infty$. There exists an Orlicz function space $L^{F}[0,1]$ with indices $\alpha_{F}^{\infty}=\alpha, \gamma_{F}^{\infty}=\gamma$ and $\beta_{F}^{\infty}=\beta$ such that $L^{p}$ is lattice-isomorphic to a sublattice of $L^{F}[0,1]$ for every $p \in\left[\gamma_{F}^{\infty}, \beta_{F}^{\infty}\right]$.

Thus for these spaces $L^{F}[0,1]$ we have $P_{F}=[\gamma, \beta] \subset[\alpha, \beta]$.

Given an Orlicz function space $L^{F}[0,1]$ such that $L^{F}[0,1] \underset{\sim}{\supset} L^{p}$, the canonical inclusion $L^{p}[0,1] \subset L^{F}[0,1]$ is always disjointly strictly singular, although the space $L^{F}[0,1]$ contains a sublattice lattice-isomorphic to $L^{p}$. The interesting case is $p=\gamma_{F}^{\infty}$ (for $p>\gamma_{F}^{\infty}$ it is easily deduced by factorization). Recall that an operator $T$ from a Banach lattice to a Banach space is disjointly strictly singular if the restriction of $T$ to any subspace generated by a sequence of disjoint functions is never invertible. Using this notion, a characterization of $L^{1}[0,1]$ among r.i. function spaces is the following ([G-H-S-S]): an r.i. function space $E[0,1]$ coincides with $L^{1}[0,1]$ if and only if for every r.i. function space $F[0,1]$ with $F[0,1] \subset E[0,1]$ and $F[0,1] \neq E[0,1]$ the inclusion $F[0,1] \subset E[0,1]$ is always disjointly strictly singular.

Let us mention also that the sets $P_{F}$ are not always closed:

TheOREM 7.2. Let $1<\alpha \leq \gamma<\beta<\infty$. There exists a $\beta$-concave Orlicz function space $L^{F}[0,1]$ with indices $\alpha_{F}^{\infty}=\alpha, \gamma_{F}^{\infty}=\gamma$ and $\beta_{F}^{\infty}=\beta$ such that $L^{F}[0,1] \underset{\sim_{\ell}}{\supset} L^{p}$ if and only if $p \in\left[\gamma_{F}^{\infty}, \beta_{F}^{\infty}\right)$.

It is an open question whether or not the sets $P_{F}$ are always convex. In other words, given $p<r<q$ and a separable Orlicz function space $L^{F}[0,1]$ with $L^{F}[0,1] \underset{\sim_{\ell}}{\supset^{p}}$ and $L^{F}[0,1] \underset{\sim_{\ell}}{\supset} L^{q}$, does it follow that $L^{F}[0,1] \underset{\sim_{\ell}}{\supset} L^{r} ?$ A similar question can be formulated for separable r.i. function spaces on $[0,1]$. Thus it would be interesting to find other classes of separable r.i. Banach function spaces on $[0,1]$ containing lattice-isomorphic copies of $L^{p}$-spaces. 
Finally let us remark that several of the above universality type results can be also stated in the quasi-Banach case. And that Kalton's result on isomorphic $L^{1}$-embeddings and Troyanski's result on isomorphic $\ell^{1}(\Gamma)$-embeddings fail to be true in this setting.

Acknowledgments. The author would like to thank L. Drewnowski, Y. Raynaud and C. Ruiz for helpful comments.

\section{References}

[A] Y. Abramovich, Operators preserving disjointness on rearrangement invariant spaces, Pacific J. Math. 148 (1991), 201-206.

[A-M-S] S. V. Astashkin, L. Maligranda and E. Semenov, Multiplicator space and complemented subspaces of rearrangement invariant spaces, J. Funct. Anal. 202 (2003), 247-276.

[B-Dc] J. J. Bretagnolle and D. Dacunha-Castelle, Applications de l'étude de certaines formes linéaires aléatoires au plongement d'espaces de Banach dans des espaces $L^{p}$, Ann. Scient. Ec. Norm. Sup. 2 (1969), 437-480.

[B-Dc-K] J. J. Bretagnolle, D. Dacunha-Castelle and J. L. Krivine, Lois stables et espaces $L_{p}$, Ann. Inst. H. Poincaré, Probab. Statist. 2 (1966), 231-259.

[C 81] N. L. Carothers, Rearrangement invariant subspaces of Lorentz function spaces, Israel J. of Math. 40 (1981), 217-228.

[C 87] N. L. Carothers, Rearrangement invariant subspaces of Lorentz function spaces II, Rocky Mountain J. Math. 17 (1987), 607-616.

[D 90] S. J. Dilworth, A scale of linear spaces related to the $L^{p}$ scale, Illinois J. Math 140 (1990), 140-158.

[D 01] S. J. Dilworth, Special Banach lattices and their applicatios, in: Handbook of the Geometry of Banach Spaces, Vol. 1, W. B. Johnson and J. Lindenstrauss (eds.), Elsevier, 2001, 497-532.

[Dr] L. Drewnowski, On symmetric basis in non-separable Banach spaces, Studia Math. 85 (1987), 157-161.

[G] J. J. Grobler, Orlicz spaces: a survey of certain aspects, in: From A. to Z. Proc. Symp. in honour A.C. Zaanen (Leiden, 1982), Math. Centre Tracts 149, Amsterdam, 1982.

[G-H] A. Garcia del Amo and F. L. Hernández, On embedding of function spaces into $L^{p}+L^{q}$, Contemporary Math. 144 (1993), 107-113.

[G-H-S-S] A. García del Amo, F. L. Hernández, V. M. Sánchez and E. M. Semenov, Disjointly strictly singular inclusions between rearrangement invariant spaces, J. London Math. Soc. 62 (2000), 239-252.

[H-K] F. L. Hernández and N. J. Kalton, Subspaces of rearrangement invariant spaces, Canadian J. of Math. 48 (1996), 794-833.

[H-P] F. L. Hernández and V. Peirats, Orlicz function spaces without complemented copies of $\ell^{p}$, Israel J. Math. 56 (1986), 355-360.

[H-R 88] F. L. Hernández and B. Rodríguez-Salinas, On $\ell^{p}$-complemented copies in Orlicz spaces, Israel J. Math. 62 (1988), 37-55.

[H-R 89] F. L. Hernández and B. Rodríguez-Salinas, On $\ell^{p}$-complemented copies in Orlicz spaces II, Israel J. Math. 68 (1989), 27-55. 
[H-R 92] F. L. Hernández and B. Rodríguez-Salinas, Remarks on the Orlicz function spaces $L^{\varphi}[0, \infty)$, Math. Nachr. 156 (1992), 225-232.

[H-R 95] F. L. Hernández and B. Rodríguez-Salinas, Lattice-embedding $L^{p}$ into Orlicz spaces, Israel J. Math. 90 (1995), 167-188.

[H-R 98] F. L. Hernández and B. Rodríguez-Salinas, Lattice-embedding scales of $L^{p}$-spaces into Orlicz spaces, Israel J. Math. 104 (1998), 191-220.

[H-R 03] F. L. Hernández and B. Rodríguez-Salinas, Lattice-universal Orlicz spaces on probability spaces, Israel J. Math. 133 (2003), 9-28.

[H-Ru] F. L. Hernández and C. Ruiz, Universal classes of Orlicz function spaces, Pacific J. Math. 155 (1992), 87-98.

[H-S] F. L. Hernández and E. Semenov, Subspaces generated by translations in rearrangement invariant spaces, J. Funct. Anal. 169 (1999), 52-80.

[H-T] F. L. Hernández and S. Troyanski, On the representation of the uncountable symmetric basic sets and its applications, Studia Math. 107 (1993), 287-304.

[J-M-S-T] W. B. Johnson, B. Maurey, G. Schechtmann and L. Tzafriri, Symmetric structures in Banach spaces, Mem. Amer. Math. Soc. 217 (1979).

[K 77] N. J. Kalton, Orlicz sequence spaces without local convexity, Math. Proc. Cambridge Philos. Soc. 81 (1977), 253-277.

[K 79] N. J. Kalton, Embedding $L^{1}$ in a Banach lattice, Israel J. Math. 32 (1979), 209-220.

[K 90] N. J. Kalton, Minimal and strongly-minimal Orlicz sequence spaces, in: Geometry of Banach Spaces, Proc. Confer. in Strobl (Austria), London Math. Soc. Lect. Note 158 (1990), 149-163.

[K 93] N. J. Kalton, Lattice structures in Banach spaces, Mem. Amer. Math. Soc. 493 (1993).

[K-T] A. Kamińska and B.Turett, Type and cotype in Musielak-Orlicz spaces, in: Geometry of Banach Spaces, Proc. Confer. in Strobl (Austria), London Math. Soc. Lect. Note 158 (1990), 165-180.

[K-M-P] A. Kamińska, L. Maligranda and L. E. Persson, Type, cotype and convexity properties of Orlicz spaces, Colloqium Dpto. Analisis. Univ. Complutense Madrid (199697), 113-126.

[K-P-S] S. G. Krěn, Ju. I. Petunin and E. M. Semenov, Interpolation of Linear Operators, A.M.S., Providence, 1982.

[K-R] M. Kranosel'skii and Ya. Rutickii, Convex Functions and Orlicz Spaces, Noordhoff, Groningen, 1961.

[Li] K. Lindberg, On subspaces of Orlicz sequence spaces, Studia Math. 45 (1973), 121146.

[Lu] W. A. J. Luxemburg, Banach function spaces, Thesis, Delft, 1955.

[L-T 71] J. Lindenstrauss and L. Tzafriri, On Orlicz sequence spaces, Israel J. Math. 10 (1971), 379-390.

[L-T 72] J. Lindenstrauss and L. Tzafriri, On Orlicz sequence spaces II, Israel J. Math. 11 (1972), 355-379.

[L-T 73] J. Lindenstrauss and L. Tzafriri, On Orlicz sequence spaces III, Israel J. Math. 14 (1973), 368-389.

[L-T 77] J. Lindenstrauss and L. Tzafriri, Classical Banach Spaces I. Sequence Spaces, Springer-Verlag, Berlin, 1977.

[L-T 79] J. Lindenstrauss and L. Tzafriri, Classical Banach Spaces II. Function Spaces, Springer-Verlag, Berlin 1979. 
[M] L. Maligranda, Indices and interpolation, Dissertationes Math. 234 (1985).

[Mu] J. Musielak, Orlicz Spaces and Modular Spaces, Lect. Notes in Math. 1034, Springer, 1983.

[M-O] W. Matuszewska and W. Orlicz, On certain properties of $\varphi$-functions, Bull. Acad. Polon. Sc. 8 (1960), 439-443.

[N] N. J. Nielsen, On the Orlicz function spaces $L^{M}[0, \infty)$, Israel J. Math. 20 (1975), 237-259.

[R] B. Rodríguez-Salinas, Subspaces with symmetric basis in the Orlicz spaces $\ell^{F}(I)$, Comment. Math. Prac. Math. 36 (1996), 201-222.

[Ra] Y. Raynaud, Complemented Hilbertian subspaces in rearrangement invariant function spaces, Illinois Math. J. 39 (1995), 212-240.

[Ra-S] Y. Raynaud and C. Schütt, Some results on symmetric subspaces of $L^{1}$, Studia Math. 89 (1988), 27-35.

[Ru] C. Ruiz, On the structure of Rosenthal spaces $X_{\varphi}$ in Orlicz function spaces, Rocky Mountain J. of Math. 27 (1997), 1223-1238.

[S 89] C. Schütt, Lorentz spaces that are isomorphic to subspaces of $L^{1}$. Trans. Amer. Math. Soc. 314 (1989), 583-589.

[S 95] C. Schütt, On the embedding of 2-concave Orlicz spaces into $L^{1}$, Studia Math. 113 (1995), 73-80.

[T 75] S. Troyanski, On non-separable Banach spaces with symmetric basis, Studia Math. 53 (1975), 253-263.

[T 90] S. Troyanski, On representation of uncountable symmetric basic sets, in: Proc. 5th. Spring Conf. of Math. Education (Gabrovo, 1976), Sofia, 1990, 169-173 (in Russian).

[Tz] L. Tzafriri, Uniqueness of structure in Banach spaces, in: Handbook of the Geometry of Banach Spaces, Vol. 2, W. B. Johnson and J. Lindenstrauss (eds.), Elsevier, 2003, $1635-1669$. 